\title{
Energy-optimal steering of transitions through a fractal basin boundary
}

\author{
Alexander N. Silchenko, Stefano Beri, Dmitrii G. Luchinsky and Peter V. E. McClintock \\ Department of Physics, Lancaster University \\ Lancaster, LA1 4YB, UK \\ a.silchenko@lancaster.ac.uk
}

\begin{abstract}
We study fluctuational transitions in a discrete dynamical system having two co-existing attractors in phase space, separated by a fractal basin boundary. It is shown that transitions occur via a unique accessible point on the boundary. The complicated structure of the paths inside the fractal boundary is determined by a hierarchy of homoclinic original saddles. By exploiting an analogy between the control problem and the concept of an optimal fluctuational path, we identify the optimal deterministic control function as being equivalent to the optimal fluctuational force obtained from a numerical analysis of the fluctuational transitions between two states.
\end{abstract}

\section{Introduction}

The stability of nonlinear multistable systems in the presence of noise, and methods allowing transitions to be steered between different co-existing attractors in such systems, are of great importance for practical applications and of obvious interdisciplinary interest. From the general point of view, there are two distinct reasons why unpredictable and complex behavior may occur in a dynamical system. The first reason is the presence of limit sets with complex geometrical structure in phase space, and the second one is complex structure of the basins of attraction, which may be fractal. Two of the most challenging and difficult problems that we face is how a fluctuational transition takes place across a fractal basin boundary (FBB), and how the same transition can be effected in the absence of fluctuations using a control force of minimum energy.

The problem of deterministic energy-optimal migration from the basin of an attractor has remained unsolved for a long time, even in the simplest case of smooth basin boundaries separating co-existing at- tractors [1]. The known approaches to the control of complex and chaotic dynamics [2] include entraining to a chosen "goal attractor" that requires large modifications of the original system's dynamics [3, 4], and a variety of minimal forms of interaction which have been restricted by the linear approximations adopted $[5,6,7]$.

A promising approach to the problem of steering transitions between co-existing stable states in the presence of an FBB is based on an analogy between the variational formulations of the deterministic control problem and the concept of the optimal fluctuational path [8]. This recent approach [9] searches for the optimal control function via a statistical analysis of the fluctuational trajectories of a dynamical system in the presence of small random perturbations. It was introduced as the result of a joint consideration of the two different kinds of problem mentioned above. The first one deals with deterministic switching from the basin of an attractor induced by an external force, whereas the second is devoted to noise-induced escape from the basin of attraction. In the limit of small noise intensity, a stochastic dynamical system fluctuates to remote states along certain most probable deterministic paths $[10,11]$, corresponding to rays in the WKB-like asymptotic solution of the Fokker-Planck equation [12]. The possibility of extending such an approach to chaotic continuous and discrete systems was established earlier $[13,14,15,16]$. The recent studies of escape in the presence of homoclinic tangencies, which are the reason for fractalization of the basins, have shown that they lead to a decrease of the activation energy [17]. However, there are still no theoretical predictions about the mechanism of escape in the case of an FBB. The problems of the uniqueness of the escape path, and of the form of the boundary conditions on the FBB, have also remained unsolved. In this paper, we show that the energy-optimal control function able to effect switching between co-existing attractors separated by an FBB can be found via a simple analysis of fluctuational trajectories. 


\section{Optimal control function}

Let us start from the general formulation of the problem under study and consider a $N$-dimensional multistable discrete dynamical system excited by a control signal $\mathbf{v}_{\mathbf{n}}$ :

$$
\mathbf{x}_{\mathbf{n}+\mathbf{1}}=\mathbf{f}\left(\mathbf{x}_{\mathbf{n}}\right)+\mathbf{v}_{\mathbf{n}} .
$$

The energy-optimal path for the control function is given by that path which minimises the following sum:

$$
\mathbf{S}=\inf _{v \in V} \frac{1}{2} \sum_{\mathbf{n}=\mathbf{1}}^{\mathbf{N}} \mathbf{v}_{\mathbf{n}}^{\mathbf{T}} \mathbf{v}_{\mathbf{n}}
$$

where $V$ is the control set of functions (control signals) that are able to move the system from one attractor to the other. The extremal problem can easily be solved by taking (1) into account by means of Lagrangian multipliers $\lambda_{\mathbf{n}}$ [15]. We thus obtain the following "Lagrangian" for minimization:

$$
\mathbf{L}=\frac{1}{2} \sum_{\mathbf{n}=\mathbf{1}}^{\mathbf{N}} \mathbf{v}_{\mathbf{n}}^{\mathbf{T}} \mathbf{v}_{\mathbf{n}}+\sum_{\mathbf{n}=\mathbf{1}}^{\mathbf{N}} \lambda_{\mathbf{n}}^{\mathbf{T}}\left(\mathbf{x}_{\mathbf{n}+\mathbf{1}}-\mathbf{f}\left(\mathbf{x}_{\mathbf{n}}\right)-\mathbf{v}_{\mathbf{n}}\right) .
$$

Further, varying $\mathbf{L}$ with respect to $\mathbf{v}_{\mathbf{n}}$ and $\mathbf{x}_{\mathbf{n}}$ we get the following $2 N$-dimensional map:

$$
\begin{aligned}
\mathbf{x}_{\mathbf{n}+\mathbf{1}} & =\mathbf{f}\left(\mathbf{x}_{\mathbf{n}}\right)+\lambda_{\mathbf{n}} \\
\lambda_{\mathbf{n}+\mathbf{1}} & =\mathbf{f}^{-\mathbf{1}}\left(\mathbf{x}_{\mathbf{n}+\mathbf{1}}\right) \lambda_{\mathbf{n}} .
\end{aligned}
$$

Equations (3) should be supplemented by the boundary conditions

$$
\lim _{\mathbf{n} \rightarrow-\infty} \lambda_{\mathbf{n}}=\mathbf{0}, \quad \mathbf{x}_{\mathbf{n}}^{\mathbf{0}} \in \text { attractor, } \mathbf{x}_{\mathbf{n}}^{\mathbf{1}} \in \mathbf{F B B} .
$$

The optimal control function $\mathbf{v}_{\mathbf{n}}^{\text {opt }}$ corresponds to a unique trajectory in phase space of (3) connecting $\mathbf{x}_{\mathbf{n}}^{\mathbf{0}}$ and $\mathbf{x}_{\mathbf{n}}^{\mathbf{1}}$ : it is that for which our energy functional $S$ takes its minimal value. This optimal trajectory can readily be found by the use of a shooting method with a properly chosen (but very small) initial vector $\left(\mathbf{x}_{\mathbf{n}}, \lambda_{\mathbf{n}}\right)$, and consequent selection of the trajectory that minimizes $S$. The paths emanating from an initial saddle point are a two-parameter set on its two-dimensional unstable manifold. In a small neighbourhood of the initial state, the manifold is a plane spanned by the two unstable eigenvectors of the linearised map (3). Initial values for the coordinates can be parameterised by the distance from the initial state and angular position; the initial values for the $\lambda_{n}$ are obtained by using the equations for the linearised manifold. During the evolution of the system (3) far from its initial state, we collect the values $S_{n+1}=S_{n}+1 / 2 \lambda_{n}^{T} \lambda_{n}$ and plot $S_{n}$ as a function of the two parameters. Thus, the global minimum of the activation energy gives us the parameters corresponding to the optimal escape path. We want

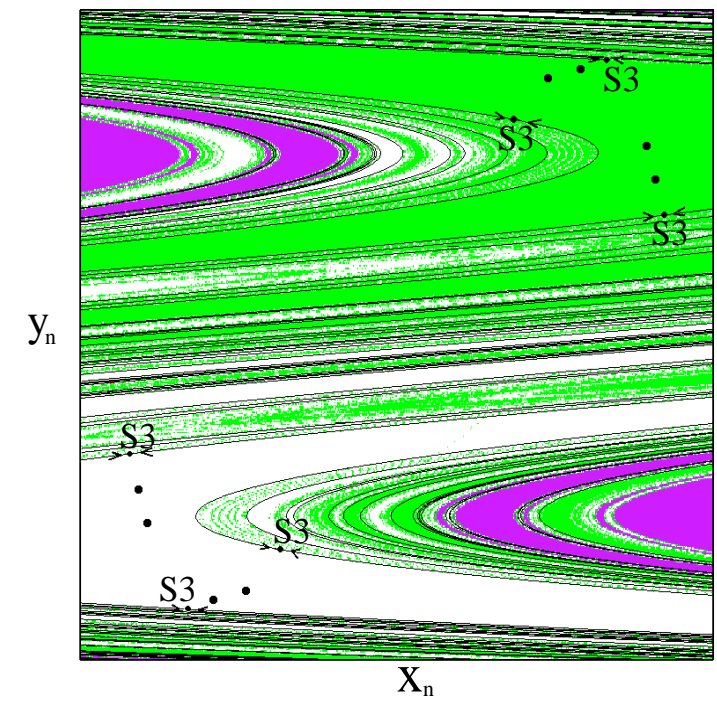

Figure 1: The co-existing stable points of period 4 (filled black circles) and their basins of attraction shown in grey and white respectively. The accessible boundary saddle points of period 3 are shown as the filled black circles $S 3$. Their stable manifolds are shown as solid black lines.

to emphasise here that the optimal trajectory is not just a mathematical abstraction. In fact, when the system (1) is driven by noise those intensity tends to zero, the escape events become exponentially rare, but they take place in an almost deterministic way following very closely the deterministic trajectory of (3).

\section{The model and results of simulations}

One of the simplest and most convenient systems of the class in which we are interested is the twodimensional map introduced by Holmes [21]. Its generic features allow us to believe that the mechanism of escape that we reveal is typical of a wide class of maps in two dimensions, and typical of many continuous systems as well. The Holmes map is

$$
\begin{aligned}
x_{n+1} & =y_{n} \\
y_{n+1} & =-b x_{n}+d y_{n}-y_{n}^{3}+v_{n} .
\end{aligned}
$$

Here $v_{n}$ is the control function. Due to symmetry, the system (5) has pairs of co-existing attractors for $b=0.2$ and $2.0 \leq d \leq 2.745$. Their basins are separated by a boundary that may be either smooth or fractal depending on the chosen parameter values. We chose for our studies $b=0.2$ and $d=2.65$, which corresponds to there being two co-existing stable points of period 4 whose basins are separated by 

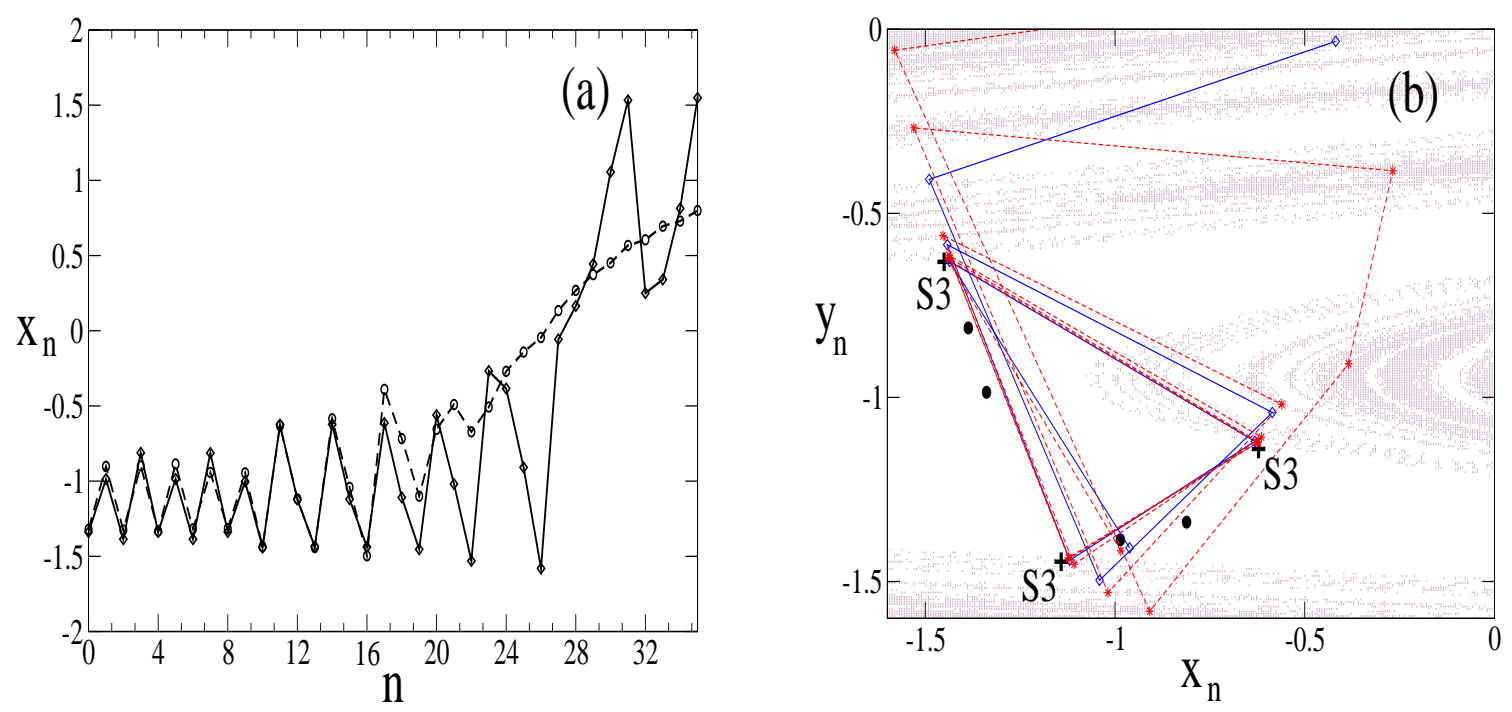

Figure 2: (a) The most probable escape path (dashed line) connecting the stable point of period 4 with the period3 saddle cycle lying on the fractal boundary obtained from the numerical simulations with $D=10^{-5}$. The optimal path found by the solution of the boundary-value problem is shown as a solid line; (b) a two-dimensional plot of the paths presented in the previous figure.

a locally disconnected FBB (see Fig. 1). The fractal dimension of the boundary has been determined numerically as $\operatorname{dim}=1.8451$ by using the "uncertainty exponent" technique introduced in [22].

Applying the above approach to (5) we get the following four-dimensional area-preserving map:

$$
\begin{aligned}
x_{n+1} & =y_{n} \\
y_{n+1} & =-b x_{n}+d y_{n}-y_{n}^{3}+\lambda_{n}^{y} \\
\lambda_{n+1}^{x} & =\left(d-3 x_{n+1}^{2}\right) \lambda_{n}^{x} / b-\lambda_{n}^{y} / b \\
\lambda_{n+1}^{y} & =\lambda_{n}^{x}
\end{aligned}
$$

In fact, the unique energy-optimal trajectory along which $S$ takes its minimal value is a heteroclinic trajectory in four-dimensional phase space connecting the stable point of period 4 with a point on the boundary. Thus, to find the optimal control function, we first need to solve the boundary-value problem, i.e. define the boundary conditions on the FBB. To find these boundary conditions and the optimal control function, we will make use of the analogy, mentioned above, between the energy-optimal control and noise-induced escape from a basin of attraction. The function $S$ in (2) coincides with the definition of the activation energy for fluctuational transitions between co-existing attractors if the control signal $v_{n}$ in (5) is replaced by white Gaussian noise such that $\left\langle\xi_{n}\right\rangle=0$ and $\left\langle\xi_{n} \xi_{m}\right\rangle=2 D \delta_{n m}$. Thus, the optimal control signal can be identified with the optimal fluctuational force which drives the system from one attractor to the other, which can be found ex- perimentally. When the system (5) is driven by noise those intesity tends to zero, it will occasionally fluctuate to the FBB following very closely the deterministic trajectory of (6). The method we used involves monitoring the system continuously and collecting all successful realizations, moving it from one stable point to the other. From these realizations, a timedependent prehistory distribution is built $[18,19]$. This distribution is characterized by a sequence of narrow spikes, as the noise intensity is decreased, allowing us to define an approximate solution for the control function. A typical optimal escape path obtained by averaging a few hundred successive trajectories, is shown in Fig. 2(a). A simple analysis of the optimal path shows that the system (5) leaves the stable point of period 4 and moves to the FBB crossing it at a point of period 3 located near, or directly on, the FBB (see Fig. 2(a)). Simple calculations have shown that a saddle point of period 3 ( $S 3$ in Fig. 1 and Fig. 2(b) with multipliers $\mu_{1}=0.001218$ and $\left.\mu_{2}=6.566269\right)$ does exist for the chosen parameter values and that it lies on the boundary. Moreover, its stable manifold (solid black line in Fig. 1) is dense in the boundary and detaches the open neighborhood including the attractor from the FBB itself, which allows us to classify it as an accessible boundary point [23].

At this stage, we know the boundary condition on the FBB, and we are ready to solve the corresponding boundary-value problem for the system (6) numerically. This can be done via a procedure involving shooting from a very small neighbourhood of the 


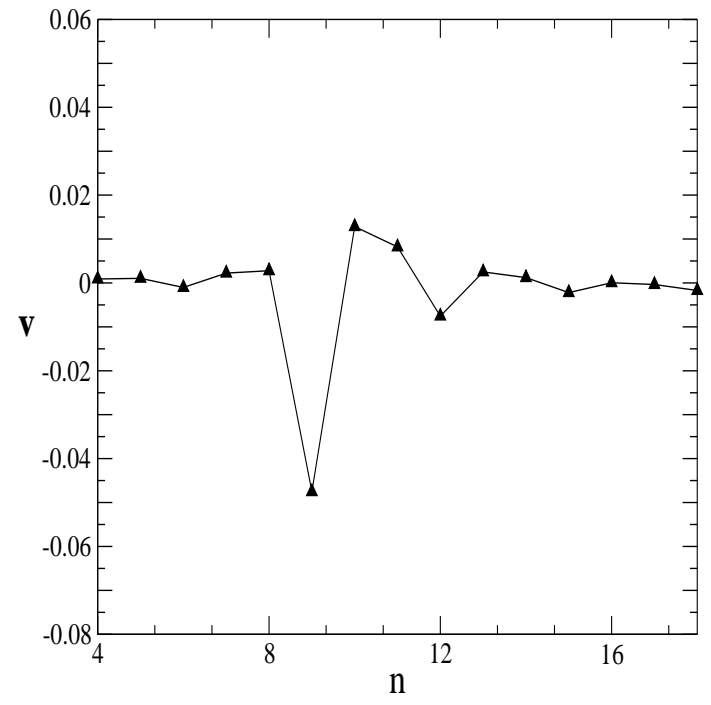

Figure 3: The optimal control function obtained from numerical simulations.

chosen saddle point, parameterizing the initial conditions as points lying on a two-dimensional unstable manifold of this saddle point, characterized by the appropriate radius $r$ and angle $\phi$, and with subsequent selection of a trajectory minimizing $S$. As clearly seen from Fig. 2, the phase trajectory in (6) along which $S$ takes its minimal value coincides with the most probable escape path obtained by taking an ensemble average of successful trajectories. Hence we can indeed identify the optimal control function with the optimal fluctuational force driving the system to the FBB. Note that no action is required to bring the system to the other attractor after it has hit the FBB, and neither is there any possibility of controlling the motion inside FBB. Our numerical calculations have shown that the control force $v(n)$ pictured in Fig. 3 really is optimal in that it minimises the energy of the control function steering the system (5) through the FBB. It was found also that the system is very sensitive to small variations in the control function: any deviation from the shape of $v(n)$ leads to a substantial increase in the energy required to perform transition.

Analysis of the structure of escape paths inside the FBB has shown that homoclinic saddle points play a key role in its formation. In the system (5), we observe an infinite sequence of saddle-node bifurcations of period $3,4,5,6 \ldots$, occurring at parameter values $d_{3}<d_{4}<d_{5}<d_{6} \ldots$ and caused by the sequent tangencies of the stable and unstable manifolds of the saddle point $O$ at $(0,0)$. The homoclinic orbits appearing as the result of these bifurcations were classified earlier as original saddles, and it was also shown that their stable and unstable manifolds cross each other in hierarchical sequence [23]. To characterize this hierarchical relationship between original saddles it is reasonable to use the pointwise dimension defined as

$$
D_{p}(S)=1-\frac{\log \lambda_{u}(S)}{\log \lambda_{s}(S)}
$$

where $\lambda_{u}(S)$ and $\lambda_{s}(S)$ are the stable and unstable eigenvalues of the Jacobian matrix of (5) at the saddle point $S$ [24]. The pointwise dimensions $D_{p}\left(S_{i}\right)$ calculated for the original saddles with periods $3,4,5,6 \ldots$ demonstrate the following hierarchical interrelation: $D_{p}\left(S_{3}\right)=1.28045, D_{p}\left(S_{4}\right)=$ $1.30294, D_{p}\left(S_{5}\right)=1.30771, D_{p}\left(S_{6}\right)=1.31825$. It is known that unstable periodic orbits embedded into chaotic saddle define a distribution of the natural measure on it both for hyperbolic and nonhyperbolic dynamical systems $[24,25]$. It is the natural measure being distributed nonuniformly on a chaotic saddle that leads to the difference in probabilities of arrival into the neighbourhoods of the different original saddles. A statistical analysis of escape trajectories has shown that these probabilities demonstrate a hierarchical interrelation [26], which is in a good agreement with the distribution of the natural measure on the chaotic saddle $O$ forming the fractal basin boundaries.

\section{Conclusions}

In conclusion, we have shown that energy-optimal control of migration between co-existing attractors is possible even where the dynamics of a system is highly sensitive to initial conditions on account of a fractality of the basins of attraction. By using a statistical analysis of fluctuational trajectories, we solved the boundary-value problem for the case of co-existing stable points separated by a locally disconnected FBB, and we found the optimal control function moving the system (5) from one attractor to the other. It was observed that the original saddles forming the homoclinic structure of the system (5) play a key role in the formation of the escape paths inside the FBB, and that the difference in their local stability defines the hierarchical relationship between them. The results obtained can be applied directly to flows having the same type of FBB, because the Holmes map was originally introduced as an approximation of the Poíncare section of the Duffing oscillator [21]. It should be noted that the existence of a locally disconnected FBB in an actual physical system was verified in full-scale experiments and that an FBB has also been observed in a discrete model of the $\mathrm{CO}_{2}$ laser [27]. 


\section{Acknowledgements}

The research has been supported by the Engineering and Physical Sciences Research Council (UK).

\section{References}

[1] L. C. Young, Lectures on the Calculus of Variations and Optimal Control Theory, Saunders, Philadelphia, 1969; P. Hagedorn, Non-linear Oscillations Claredon Press, Oxford, 1982.

[2] A. L. Fradkov and A. Y. Pogromsky, Introduction to Control of Oscillations and Chaos, Series on Nonlinear Science A, vol. 35 World Scientific, Singapore, 1998.

[3] E. A. Jackson, "The OPCL control method for entrainment, model-resonance, and migration actions on multiple-attractor systems," Chaos, vol. 7, pp. 550-559, 1997.

[4] S. P. Raj and S. Rajasekar, "Migration control in two coupled Duffing oscillators," Phys. Rev. E., vol. 55, pp. 6237-6240, 1997.

[5] T. Shinbrot, C. Grebogi, J. Ott and E. Yorke, "Using small perturbations to control chaos," Nature (London), vol. 363, pp. 411-417, 1993; D. Auerbah, C. Grebogi, J. Ott and E. Yorke, "Controlling Chaos in High Dimensional Systems," Phys. Rev. Lett., vol. 69, pp. 3479-3482, 1992.

[6] B. Hübinger, R. Doerner, W. Martienssen, M. Herdering, R. Pitka, U. Dressler, "Controlling chaos experimentally in systems exhibiting large effective Lyapunov exponents," Phys. Rev. E., vol. 50, pp. 932-948, 1994.

[7] E. Barreto, E. J. Kostelich, C. Grebogi, J. Ott and E. Yorke, "Efficient switching between controlled unstable periodic orbits in higher dimensional chaotic systems," Phys. Rev. E., vol. 51, pp. 41694172, 1995.

[8] W. H. Fleming, "Exit probabilities and stochastic control," Appl. Math. Optim., vol. 4, pp. 329-346, 1978; P. Whittle, Risk-sensitive optimal control, Wiley, Chichester, 1990; V. N. Smelyanskiy, M. I. Dykman, "Optimal control of large fluctuations," Phys. Rev. E., vol. 55, pp. 2516-2521, 1997.

[9] D. G. Luchinsky, I.A. Khovanov, "Fluctuationinduced escape from the basin of attraction of a quasiattractor," JETP Lett., vol. 69, pp. 825-830, 1999; I. A. Khovanov, D. G. Luchinsky, P.V.E. McClintock, and R. Mannella, "Fluctuations and the energy-optimal control of chaos," Phys. Rev. Lett., vol. 85, pp. 2100-2103, 2000.

[10] L. Onsager, and S. Machlup, "Fluctuations and irreversible processes," Phys. Rev., vol. 91, pp. 1505-1512, 1953.
[11] M. I. Dykman, P. V. E. McClintock, V. N. Smelyanskiy, N. D. Stein, and N. G. Stocks, "Optimal paths and the prehistory problemfor large fluctuations in noise driven systems," Phys. Rev. Lett., vol. 68 2718-2721, 1992.

[12] M. I. Freidlin and A. D. Wentzel, Random Perturbations in Dynamical Systems, (Springer, New York, 1984).

[13] R. L. Kautz, "Activation energy for thermally induced escape from a basin of attraction," Phys. Lett. A, vol. 125, pp. 315-319, 1987.

[14] P. D. Beale, "Noise-induced escape from attractor in one-dimensional maps," Phys. Rev. A, vol. 40, pp. 3998-4003, 1989.

[15] P. Grassberger, "Noise-induced escape from attractors," J. Phys. A: Math. Gen., vol. 22, pp. 32833290, 1989 .

[16] R. Graham, A. Hamm, and T. Tel, "Nonequilibrium potentials for dynamical systems with fractal attractors or repellers," Phys. Rev. Lett., vol. 66, pp. 3089-3092, 1991.

[17] S.M. Soskin, M. Arrays, R. Mannella, and A.N. Silchenko, "Strong enhancement of noiseinduced escape by nonadiabatic periodic driving due to transient chaos," Phys. Rev. E., vol. 63, pp. 051111-6, 2001.

[18] D. G. Luchinsky, R. S. Maier, R. Mannella, P.V.E. McClintock, D. L. Stein, "Experiments on critical phenomena in anoisy exit problem," Phys. Rev. Lett., vol. 79, pp. 3117-3120, 1997; D. G. Luchinsky, "On the nature of large fluctuations in equilibrium systems: observation of an optimal force," J. Phys. A, vol. 30, pp. L577-583, 1997; D. G. Luchinsky and P. V. E. McClintock, "Irreversibility of classical fluctuations studied in analogue electrical circuits," Nature, vol. 389, pp. 463466, 1997.

[19] D.G. Luchinsky, P.V.E. McClintock, and M.I. Dykman, "Analogue studies of nonlinear systems," Rep. Prog. Phys., vol. 61, pp. 889-997, 1998.

[20] S.W. McDonald, C. Grebogi, E. Ott, and J.A. Yorke, "Fractal basin boundaries," Physica, vol. 17D, pp. 125-153, 1985.

[21] P. Holmes, "A nonlinear oscillator with a strange attractor," Phil. Trans. R. Soc., vol. A292, pp. 419-448, 1979.

[22] C. Grebogi, S. W. McDonald, E. Ott, and J. A. Yorke, "Final-state sensitivity - an obstruction of predictability," Phys. Lett., vol. 99A, pp. 415-418, 1983.

[23] C. Grebogi, E. Ott, and J.A. Yorke, "Basin boundaries metamorphoses - changes in accessible 
boundary orbits," Physica, vol. 24D, pp. 243-262, 1987.

[24] C. Grebogi, E. Ott, and J.A. Yorke, "Unstable periodic orbits and the dimensions of multifractal chaotic attractors," Phys. Rev. A, vol. 37, pp. 1711$1724,1988$.

[25] M. Dhamala and Y.-C. Lai, "The natural measure of nonattracting chaotic sets and its representation by unstable periodic orbits," Int. J. Bif. Chaos, vol. 12, pp. 2991-3005, 2002.

[26] A. N. Silchenko, D. G. Luchinsky, and P. V. E. McClintock, "Noise-induced escape through a fractal basin boundaries," Physica A, to be published.

[27] V. N. Chizhevsky, E. V. Grigorieva and S. A. Kashchenko, "Optimal timing for targeting periodic orbits in a loss-driven $\mathrm{CO}_{2}$ laser," Opt. Commun., vol. 133, pp. 189-195, 1997. 